Research Article

Vít Pokorný*

\title{
Polyrhythmic Arrangements: Rhythm as a Dynamic Principle in the Constitution of Environments
}

https://doi.org/10.1515/opphil-2020-0192

received May 6, 2021; accepted November 16, 2021

\begin{abstract}
This study explores the concept of rhythm and the relation of rhythm to the environment. Rhythm is not conceived of simply as a linear sequence of beats and pauses, but as a formative dynamic principle operating in all living systems. Following the rhythmanalysis of H. Levebvre and C. Regulier, phenomenological analyses of rhythm in Schutz and Richir, and a deleuzian processual approach to rhythm and milieu, this study attempts to address rhythm in terms of polyrhythmic bundles, which may be in states of eurhythmia or arrhythmia. Specifically, the study focuses on three types of polyrhythmic arrangements individual organism, garden, and city, on which it shows several types of rhythmic formations and rhythmic principles, whose interplay or discrepancy determines the formation of distinct types of living environment.
\end{abstract}

Keywords: rhythm, rhythmanalysis, dynamic patterns, polyrhythmic arrangements, environment

\section{Prelude}

Before considering the concept of rhythm itself, let me comment on the concept of noise used by M. Serres.

Noise is the pure chaos, the pure undifferentiated, totally symmetrical furry, the pure multiplicity our of which everything emerges like Aphrodite out of the sea. Noise cannot be a phenomenon, he says, because every phenomenon is separate from it ... All patterns, any notion of order - classes, genera, concepts, unities, entities - emerge out of noise; and this noise, this pure multiplicity is time - or rather time, he says, is a threshold between disorder and redundancy, it is the multiplicity next to chaos and prior to all spatialities. ${ }^{1}$

In the following text, I will attempt to show that contemporary rhythm studies do not comprehend rhythm just a sequence of beats but more generally as an organizing pattern or principle that is fundamental to the constitution of all living forms and their environments as well as to the constitution of phenomena in time. Since rhythm is a type of order, type of arrangement based on intertwining between difference and repetition, it is, ontologically speaking, preceded by noise which comes before and after any notion of order. This pure multiplicity is certainly nothing static, it is a pure flow, an escape without return, a formless chaos within which nothing repeats itself and anything disappears as soon as it had appeared. There is no place, no direction, no identity, no memory.

1 Herzogenrath, Time, 34.

\footnotetext{
* Corresponding author: Vít Pokorný, Institute of Philosophy, Czech Academy of Sciences, Praha, Czech Republic, e-mail: ptvok23@gmail.com
}

Ә Open Access. () 2021 Vít Pokorný, published by De Gruyter. (cc)BY This work is licensed under the Creative Commons Attribution 4.0 International License. 
If we understand being as happening, as becoming, if it is processual than it needs a principle of order, a principle that allows for constitution of duration. This principle that emerges within the apeiratic noise is, metaphorically speaking, a vortex, i.e. an interruption of the flow and folding in the sense of turning around itself. Within a pure flux, vortices form themselves, they self-organize as they revolve around themselves so that they can endure for certain time.

Depending on its intensity, the vortex can condense, solidify, temporarily settle, and then dissolve again in the continuity of the current. Rhythm first emerges as the difference between flowing and stopping and consists of backward movement, repetition, interruption of flow that folds in on itself.

\section{Concept of rhythm}

This preliminary speculative intuition is methodological in nature, it characterizes the initial general perspective, which must be substantiated by descriptions of the embeddedness of rhythm in the environment. However, right at the beginning, such description encounters a fundamental obstacle since rhythm cannot be immediately given in perception. Rhythm cannot be directly seen, heard, touched, smelled, or tasted, yet all types of perceptual phenomena can be rhythmic. Also, rhythm is not just an abstraction but a discernible part of what appears. It is, however, never given separately as it always appears as rhythm of something. Regarding the sonic thinking and phenomenology of sonic environments, I would like to stress that rhythm is not sonic in nature, nor visual, tactile, olfactory, or gustatory. As A. Schutz puts it: "It is not at all certain that rhythm is essential to musical experience itself." ${ }^{2}$ Rhythm transcends perceptual phenomena, yet at the same time it is always part of their structure.

Phenomenologically speaking, rhythm determines the very structure of perception. According to W. Sethares, ${ }^{3}$ the process of perception is necessarily related to regularity and continuity of something that is perceptually present, but it also goes beyond the immediate presence and aims for the future regularity. Rhythm defined as the relation between difference and repetition thus "imposes temporal structure on cognition." ${ }^{4}$ Also, according to $\mathrm{M}$. Richir, ${ }^{5}$ perception has rhythmic structure already on the level of passive synthesis, on the level of pre-predictive constitution of phenomenality. To be given in perception, a phenomenon cannot consist of purely temporal sequence of timeless moment. A musical phenomenon is not given as a sequence of pure separate tones, but as a global presence, which is the unity of the retention of what is no longer there and the protention of what is not yet. This global presence holds together thanks to the resonance between these time elements that are connected at a distance, which means to Richir that the time field of the phenomenon is spatial from the beginning, that there is spatiality within temporality. A phenomenon is thus established as a rhythmic repetition of before, now, and then, and only thanks to this rhythm does it acquire its non-conceptual coherence. Such rhythm thus articulates the unformed sensory matter and holds the phenomenon together. Each phenomenon is thus fundamentally rhythmic already on the pre-predictive level of passive synthesis, when its elements are repeated, when they return and revolve around each other in the process of their own disappearance. Interruption, return, and repetition are thus already present within the pure flux of time.

Even at this phenomenological level, we see that rhythm cannot be described otherwise than as rhythm of something, as a spatio-temporal relation between different "beats" of time. More so, it applies for the level of established phenomena where rhythm is always rhythm of something - respiration, growth and withering, alteration of steps, change of seasons, energy intake and output, alteration of day and night, switching on and off city lights, or regularity of heartbeat.

2 Schutz, "Fragments," 261.

3 Sethares, Transforms, 96.

4 Ibid.

5 Richir, "Pasivní Syntéza.” 
In fact, contemporary rhythm studies recognize and study many different types of rhythmic phenomena, both natural and cultural. Concept of rhythm pertains to a vast range of various phenomena. Chronobiology recognizes three basic rhythm domains - circadian, ultradian, and infradian ${ }^{6}$ within which all the regular, repeating, and self-organizing cycles, clocks, and oscillations of life appear. Biological and physiological explorations of rhythm have so far uncovered and described countless types of rhythmic phenomena on the environmental, organic, or molecular levels through which life sustains and organizes itself in time. ${ }^{7}$ Beside these natural rhythms, our social and cultural lives are also organized rhythmically whereas social rhythms ${ }^{8}$ on the one hand depend and relate to the natural ones, but on the other hand constitute new rhythm domains that may become independent or even contrary to the biological ones. In this regard, B. Adam stresses that although the duality of nature and culture has no meaning from a temporal perspective, we must after all "recognize important distinction between cultural time(s) and the temporalities of nature while appreciating their mutual interpenetration and influence." 9

My approach to the problem of rhythm focuses on the mutual interpenetration and influence between various types of rhythms that co-constitute different environments whereas environment might be described as a scape (a soundscape, a rhythmscape, timescape, etc.) which consists in intertwining of individual rhythms that constitutes environments as dynamic polyrhythmic bundles or arrangements. Such approach is not new as it is based on the rhythmanalysis first formulated by H. Lefebvre and C. Regulier ${ }^{10}$ and further elaborated by my scholars like B. Adams, T. Edensor, R. Smith and K. Hetherington, F. Neuhaus, or F. Wunderlich, yet this text is my first serious encounter with the field and preparation for further research. Second key point of my approach is that the concept of rhythm consists in, as every concept, co-dependence between various layers of its meaning. Although we cannot define rhythm simply as an abstract linear sequence of consecutive pulses, the linear rhythm is still useful in the attempt to understand the place of rhythm in the environment. I found a suitable definition of linear musical rhythm in J. Grahn's article. According to her, "rhythm is defined as the pattern of time intervals in the sequence."11 Rhythmic patterns comprise onset of a stimulus and intervals between them in a sequence. Musical rhythm is thus a series of beats (stimulus) and periods of their repeats whereas a shorter period between beats means faster tempo.

This definition of linear or musical rhythm offers several important motives. First, the rhythm is a pattern, i.e. as a sequence of beats and pauses between them. The uninterrupted sound has no rhythm. For a rhythm to occur, the continuous duration must be interrupted, and it must return and continue so that it can be interrupted again. The relationship between beats and gaps is called the tempo, which determines the speed of the rhythm. The longer the beat and the pause, the slower the tempo. Any given rhythm is then given by the whole sequence, the overall pattern of interconnected micro-events - beats. The essential part of the given rhythm is the regularity of the repeated return of the same, although a pulse, or a beat is never the same. Rhythmic repetition means that what comes back always comes as something new, but at the same time as something that repeats itself. Yet, such formal characteristics are not enough to explain how the rhythm is embodied in the lived environment as not just temporal but also as spatial phenomenon.

It is thus inaccurate to understand rhythm only based on a simple notion of drumming, i.e. as a sequence of successive beats. The very rhythm of drumming is derived from previous rhythmic occurrences, it is a human appropriation of the predominant rhythms of bodies and environments. Drumming is a deterritorialized rhythm, techné, nature transformed into culture, an imitated element of the living world transformed into an artefact. And quite contrary to our idea of isolated musical rhythm, naturally occurring rhythms never come alone. Each environment comprises multiple rhythms arranged as a rhythmic

\footnotetext{
6 Koukari and Sothern, Introducing Biological Rhythms, 6-11.

7 See Palmer, An Introduction.

8 See McNeill, Keeping Together; and Gosden, Social Being.

9 Adams, Timescapes, 13.

10 See Lefebvre and Regulier, Rhythmanalysis.

11 Grahn, “Neural Mechanisms,” 586.
} 
"bundles, bouquets or garlands."12 In the following text, I will therefore focus on the description of polyrhythmic bundles, which are characterized by the reciprocal co-determination of various rhythms.

Deleuze and Guattari state in this connection: "From chaos, Milieus and Rhythms are born ... Every milieu is vibratory ... a block of space-time constituted by the periodic repetition of the component ... Every milieu is coded, a code being defined by the periodic repetition." ${ }^{13}$ Rhythm and environment are interconnected, coextensive, unfolding simultaneously. Each rhythm is composed of different components, a different and otherwise long type of pulse and an otherwise long type of gap between them. For example, the heartbeat, which is indirectly accessible as a sound event, involves contraction and expansion of the heart muscle which is immediately imperceptible for itself, and persists in the background within the polyrhythmic system of the whole organism (an organic milieu) which maintains itself in mutual codetermination with myriads other rhythms within the wider environment. Obviously, living environments are formed not just by linear rhythmic sequences but also by cyclic rhythmic patterns. As Lefebvre states: "Everywhere where there is interaction between a place, a time, and expenditure of energy, there is rhythm. Such premise identifies repetition of movement and action, the entanglement of linear and cyclical rhythms and phases of growth and decline." 14 "Every rhythm implies the relation of a time with space, a localized time, or if one wishes, the temporalized space." 15

Edensor thus does not define rhythm simply as a sequence in time. Each rhythmic sequence is bound to an action and movement, to the continuous repetition of the pulse that entails expenditure of energy. In other words, rhythm is originally embodied, it is not just a time sequence. Even beforementioned drumming is not simply sequence of acoustic beats and pauses. It is also a sequence of the drummer's movements associated with the release of energy. The linear rhythm thus involves the movement and action of an organism or machine and is associated with the release and, of course, the supply of energy. The energy consumption itself is also rhythmic in nature, the output presupposes income. Each rhythm involves the transformation of energy through the movement of bodies. The rhythm is thus tied to the presence of a living system that moves by itself within an environment.

Indeed, persistence of an environment requires continuation of various rhythmic processes. Death as a cessation continues with decay and disintegration, which itself constitutes a part of the cyclic rhythms of the environment. Cyclic rhythms are global in nature. The cyclic rhythm of sleep and waking is linked to the alternation of day and night and thus to the rhythm of the movement of the Sun and the Earth, to the phases of the Moon, and to the rhythmic alternation of weather. Cyclic rhythms are of a different nature than linear ones. They do not involve alternating pulses and pauses but alternation of individual intersecting phases. Interruption is a part of them as the end of one phase and the occurrence of another. The end of the growth phase of an annual plant ends with flowering, followed by sowing, withering, and disintegration of the plant body. The night phase follows the day phase, but there is no pause or gap between the two, but a gradual dimming transition. The cyclic rhythm is therefore continuous, never silent, it does not repeat elementary pulses, but mutually overlapping phases.

In both cases, however, it is true that "rhythm and schema are ways to establish order or to form an arrangement." 16 Tosaki states that E. Benveniste drew attention to the relationship between rhythm and form in his research whereas Greek rhythmos can be interpreted as an instantiation of a form, namely the type of form "in the instant that is assumed by what is moving, mobile or fluid, the form of that which does not have organic consistency." ${ }^{17}$ It could be, for example, a shape of a liquid element, arbitrarily modified robe, or a current mood. Thus, in its oldest meaning, rhythm has not much to do with repetition and return, but with a random arrangement into some transitional form. Only later, first with Plato, did rhythm become a measure of movement, and later it was associated with a linear musical concept by Aristoxenus who states

12 Lefebvre and Regulier, Rhythmanalysis, 20.

13 Deleuze and Guattari, A Thousand Plateaus, 313.

14 Lefebvre and Regulier, Rhythmanalysis, 15.

15 Lefebvre, On Cities, 230.

16 Tosaki, Modrian, 148.

17 Ibid., 144. 
that "we must recognize rhythm and the rhythmizable medium as separate notions and separate natures, related to one another in the same kind of way as shape (schema) and shapable material." ${ }^{18}$ Such divorce of rhythm from rhythmizable material inevitably turned rhythm into a definable and measurable formal property of linear sequence. Yet, from the embodied and situated perspective, there is no separable rhythmic form that imprints its structure to the material. Instead, rhythm is conceived of as a principle inherent in the processes of self-constitution of living forms in their environments. Also J. Wellman shows in her book on the biology and epistemology of rhythm that rhythm cannot be defined by mere periodicity of division. From her perspective, rhythm is far more than mere sequence since succession or sequence as such are meaningless and accidental while rhythmic order means that the accidental nature of sequence is transformed into necessity, "whereby the whole is no longer subjected to time but rather possesses time within itself."19

Yet, what does it mean that the rhythmic whole possesses time within itself? A random sequence of consecutive events is not yet a rhythm. Rhythm is established only as a meaningful order. But under what circumstances and how does a random sequence turn into a meaningful order? Consider the case of the rhythm of walking. The rhythm of walking is not just an accidental sequence of steps. The walking acquires a rhythm only as a hurried gait, as a fast run of a beast chasing its prey, as a slow and prudent step of a mountaineer, or as a rehearsed marching step of a military unit. The rhythmicity of the steps is thus necessarily connected with the direction of the walker, with his intention and with the overall context of his movement. Rhythm, in this respect, is not a random sequence, but an organizing pattern that is not given from the outside but is a principle of self-organization of event timing itself in space. Space-time ceases to be chaos as it organizes itself through rhythmic events. Self-organization here means a relation to oneself, a feedback principle, the spiralling duration of a whole.

Also, in Whitehead's An Enquiry concerning the Principles of Natural Knowledge rhythm represents a way of identifying living object. Whitehead even states that

wherever there is some rhythm there is some life and rhythm is therefore coterminous with life whereas rhythm has a particular structure. It involves a pattern and to that extent is always self-identical. The rhythmic quality of an event depends also upon the difference involved in each exhibition of the pattern. The order of rhythm is therefore a structure of deviating repetition; it is always the fusion of sameness and novelty. ${ }^{20}$

Each inhale is always a new inhale, the pattern of inhale and exhale is always repeated as the same, and it is always something new. The rhythm of the breath constitutes identity of duration, it carries a continuing duration in itself. It is a duration that is open to the repetition of the same event, which is always different and keeps coming back. It develops as a succession of constantly new pulses of the same breathing organism, as an alternation of filling and emptying.

\section{Organic rhythms: Organisms and milieus as a polyrhythmic arrangement}

As was already stated before, rhythm as a form of self-expression and self-constitution of the living never occurs alone. Every living system, whether it is an individual organism or an entire milieu, represents a complex polyrhythmic bundle. In the following analyses, I will try to describe three types of polyrhythmic bundles: an individual organism, a garden as a set of living organisms, and a city as a system of organic and technological rhythms.

18 Aristoxenus, Elementa, 3.

19 Wellman, Becoming, 86.

20 Ibid., 24-5. 
Every individual organism contains at least three different layers of rhythmic activity: physiological processes of metabolism and respiration, embryological processes of development, and processes of metamorphoses. ${ }^{21}$ Each of these rhythmic layers is even more complex, since it includes other levels of rhythmic self-organization. Global rhythms of metabolism, respiration, and pulsating oxygenation of the blood presuppose myriad cellular and molecular cycles such as rhythmic switching on and off neural circuits, chemical and mechanical decomposition of food into usable resources, sedimentation and gradual release of substances, and countless others. At the same time, every living organism goes through the rhythm of its own self-transformation. An annual plant passes from seed through germ to stem and leaf formation, from flowering to fruit formation and seed release, and finally to decomposition. The formation of each organism from the initial embryo was described ... as the gradual emergence of forms out of initially homogenous organic matter which is based on rhythmic principles. Wellman here refers to the work of C. F. Wolff, the founder of modern embryology, who states that every organism's rhythmic arrangement can be described as an "interplay between three dynamics - repetition, pulsation, and spiral motion." 22 In this framework, repetition concerns process of nutrition and growth during individual's entire lifetime. Pulsation concerns a rhythmic relation between fluid and static states, whereas formation of every organic structure requires both - flow of nutrients which allows for the formation of a static structures. The spiral motion designates the process during differentiation within the embryo when an undifferentiated system of organs differentiates itself into specialized parts.

This unsystematic description which is too far from being complete ${ }^{23}$ gives us at least a glimpse of individual organism's polyrhythmic complexity. For such a polyrhythmic bundle to last in its duration, it must be able to maintain the still endangered state of eurhythmia. Lefebvre defines eurhythmia as a situation when "the eurhythmic body, composed of diverse rhythms - each organ, each function, having its own - keeps them in metastable equilibrium." ${ }^{24}$ It is a situation when all the rhythms unite or cooperate in a state of health, be it health of an organism or whole environment, biological or social body. Eurhythmia is a continuous state of dynamic equilibrium, an open balance between the rhythms of the organism itself and the surrounding environment in which it can live. The counterbalance of eurhythmia is arrhythmia, i.e. discordance or desynchronization of rhythms, a situation when polyrhythmia breaks apart resulting in conflict and illness. However, the duration of health or the onset of illness is not just an internal situation, because all basic organic rhythms depend on the connection of the organism with the environment, on mutual codetermination within a wider whole, which can be, for example, a garden.

The garden can be understood as a milieu formed by a complex of rhythmic patterns that create complex songs and melodies that interact and resonate with each other. The garden is composed of individual organisms and their interrelationships - plants, trees, birds, insects, fungi, and domestic animals. These organisms can only thrive when the elemental rhythms of the whole environment work the rhythms of soil, water, air, and light with their drying and humidification, lighting and dimming, warming and cooling, settling and dissolving. Obviously, these rhythms are tied to the earth's rhythms - alternation of day and night, seasons, phases of the moon, seismic flows of the magnetic field, etc., which are all related to the cyclically changing position of the Earth rotating within the solar system..$^{25}$

However, the garden is not a wild autonomous environment. It is a place that serves people and as such is territorialized by human activity - planting and harvesting, processing plant residues through composting, building flower beds, fences, and paths, irrigating and weeding, pruning trees, regular care for domestic animals, and much more. Healthy - growing, fruiting, sounding, and smelling - garden requires the harmonization of human intention with the autonomous processes of individual organisms and the whole environment. The listening gardener is the one who provides space for the thriving development of

21 Ibid., 95.

22 Ibid., 96-7.

23 For the complete overview of organisms biological rhythms see, for example, Koukkari and Sothern, Biological Rhythms.

24 Lefebvre and Regulier, Rhythmanalysis, 20.

25 See Steiner, Agriculture Course. 
intertwined rhythmic patterns of other organisms, which in turn can provide him with nutritional, aesthetic, or economic resources for his own well-being. Gardens can surely suffer from diverse types of natural arrhythmia - floods or droughts, plant diseases or invasive organisms. Yet, the fundamental conflict is always the conflict between human territorial activities and natural rhythmicity of the milieu, between too much order and no order, between total human technological control over the territory and complete lack of care. The first results in creating a sterile garden with isolated decorative plants in concrete beds and monoculture lawn periodically decimated by machinery, while the second results in the reversal of the garden back to the wild state of uncontrolled growth and decay.

\section{The city: Urban rhythms and cracked bundles}

When explaining the difference between the natural and cultural rhythms B. Adam states that "the time structure associated with gardening are closely tied to the rhythms and temporalities of the seasons, weather, plants and soil and thus bear little resemblance to the invariable, standardised, universal time of clocks and calendars." ${ }^{26}$ According to her, gardeners recognize that there is a right time for every activity and every organism whereas the rightness of the time is always context dependent. Rhythmicity of urban and industrial environment is different, it creates new, artificial rhythmic layers and relations which tend to overrun and overturn the natural ones. The right time is no longer context dependent; it depends on the will of urban consumers and industrial producers.

Garden is certainly not independent of cultural production, in fact represents one of the first cultural forms if we understand culture as a specific way of human adaption, namely, an adaptation that consists in transformation and control of the environment. Deliberate, organized, and social human activity brings new rhythms and intensities, sequences and tempos into the environment, new forms of time-space organization that transform the whole environment so that it becomes a cultural environment. Culture arises, besides other activities, in the acts of cultivation and fencing, in the creation of gardens and cities. According to M. Eliade, both acts are an imitation of the original divine act of creation, which consists in the transformation of chaos into the cosmos, in the transformation of unorganized and disordered spontaneity into the form of order.

When the Scandinavian colonists took possession of Iceland, Landnáma, and began to cultivate it, they regarded this act neither as an original undertaking nor as human and profane work. They enterprise was for them only the repetition of a primordial act: the transformation of chaos into the cosmos by the divine act of Creation. By cultivating the desert soil, they in fact repeated the act of gods, who organized chaos by giving it forms and norms. ${ }^{27}$

The garden as the giving of shapes and norms begins with the removal of part of the space from the power of the forest that continues to be maintained by regular cultivation. The city begins by laying a boundary around a system of gardens and dwellings. The original ancient city is determined by its walls, as in the case of Uruk from the epic of Gilgamesh: "Ur-shanabi, climb Uruk's wall and walk back and forth! Survey its foundations, examine the brickwork! Were its bricks not fired in an oven? Did the Seven Sages not lay its foundations? 'A square mile is city, a square mile date-grove, a square mile is clay-pit, half a square mile the temple of Ishtar: three square miles and a half is Uruk's expanse."”28

The founding of the city does not consist only in the enclosing of space, but also in starting a new time cycle, a new system of regularly recurring human activities - production and construction (bricks made of burnt clay), gardening (the date-grove), mining (the clay-pit), religious rituals and festivities (Ishtar's temple), and education (seven sages - recurring and regular transmission of cultural memory from

26 Adam, Timescapes, 95.

27 Eliade, Myth, 10.

28 The Epic of Gilgamesh, 99. 
generation to generation). Ancient and medieval cities are cities of walls, cities - states, cities - fortresses. They are surrounded by the agricultural countryside that serves for their further growth and by uncontrolled territories beyond the reach of human power, by forests full of monsters, game, and other inhuman beings. Cities are islands of order in the seas of chaos that pulsate with their own artificial life. As centres of crafts, trade, wealth, and cult, they draw resources from their surroundings and produce energy that they expel.

M. Marcelli describes this dynamism as the rhythmic encounter of openness and closedness. Medieval cities are centres of trade and as such absorb human and material resources from the countryside and transform them into the city walls and urban activities. Regularly recurring markets "open the city gates and bring streams of people." 29 Such openness which also includes exchange of information and city's expansion is compensated by the contrary principle of closedness. A city, surrounded by its walls, creates an autonomous, self-determining space-time and as such is related to the protection of territory and maintaining its own identity while at the same excluding foreign elements.

Yet, this old and original city form had rapidly disappeared with the development of military technologies and later with the advent of industrial revolution. Cities lost their walls and expanded into the landscape, gradually transforming formerly uninhabited terrain into industrial landscape. The basic urban rhythms of closing and opening had been intensified. The city turned into an industrial-mechanical framework and the pace of its growth, production, exchange of goods, energy, people, and information grew exponentially. The city was transformed into a giant machinery whose life unfolded according to the rhythms of the mechanical clocks and the speed of all its engines. ${ }^{30}$ The city becomes a carefully planned and constructed system of various interrelated technological devices and social apparatuses. The streams of water that flow inside the city and are constantly consumed and need to be constantly replenished are coupled with the streams of electricity produced by constantly rotating energy generators. These streams combine again with cycles of information production whose aim is to control all urban activities, including streams of vehicles that increase or decrease depending on the social and biological rhythms of its inhabitants.

In the post-industrial, globalized civilization even this layer of modern mechanical cities has changed. Smith and Herrington therefore point out that "rhythms that emerged from and drove change in Engel's Manchester and Park's Chicago - mass industry and the State - have not simply accelerated but, rather, have dissipated." ${ }^{31}$ This dissipation process can be interpreted in at least two ways. The first is that the rhythms of the mechanical cities have reached the peak of their intensity. They are overcrowded, they can no longer accommodate more inhabitants, they expand further and further into the landscape in the form of suburbs and satellites, they crumble and disintegrate, their flows become clogged and interrupted, their arrhythmias grow. At the same time, the boundaries between the city and non-city have disappeared as we can see in the case of the world's megacities, giant agglomerations, and conurbations. "The machinic and generalized, dominating and resisting rhythms of industrial city are seen to give way to emergent and competing rhythms which are at once more closely tied to specific sites whilst being increasingly intertwined with global flows of ideas, people and objects.” ${ }^{2}$ The city as a closed entity thus disappears, dissolves, and spills over into the landscape. It can no longer be captured as a clearly defined whole, strictly separated from the village and from the landscape. All human dwellings are interconnected by networks of communications both with each other and with sites that serve as sources of food, raw materials, or leisure. The urban environment has also ceased to be purely mechanical and built of inorganic materials (solidification of concrete, asphalt, ferro-concrete, glass, etc.), it is interwoven with parks, urban greenery, gardens, or wild areas of brownfields.

It is no longer possible to overlook and control the city and grasp all its basic rhythms. Also, we can no longer strictly distinguish it from the natural rhythms of the former nature which is present in the city in at

29 Marcelli, Filozofi, 22.

30 See Neuhaus, Emergent Dimensions.

31 Smith and Hetherington, "Urban," 5.

32 Ibid., 6. 
least two ways: in the human, plant, and animal bodies of its inhabitants, and in the processes of transformation of the urban environment itself, in the processes of disintegration and extinction of entire streets and neighbourhoods, in the processes of dissolving the identity of today's cities due to pulsating currents of tourist, the activities of development companies, or the tumour swelling of contemporary metropolises such as the South American favelas or the slums of Indian cities.

Instead of previous cities, we are therefore dealing with "new assemblages of rhythmic practices, new spatio-temporal arrangements and disconnections demanding new forms of urban citizenship, use, and indeed, critical engagement." ${ }^{33}$ Therefore, instead of describing the rhythmic structure of the city as a whole, some authors who develop Lefebvre's original insights ${ }^{34}$ propose a description of these rhythmic practices associated with (1) the everyday life of our socio-cultural ones: This includes a description of daily, weekly, monthly, and annual cycles associated primarily with the alternation of work, entertainment, and leisure; (2) the rhythms of the physical or biological plane, which includes sleep and wakefulness, food, sexuality, birth and death, adolescence, and aging, and much more; (3) the rhythms of specific environments such as e.g. railway stations, airports, factories, power plants, shops, restaurants, sports grounds, offices, hospitals, schools, industrialized fields, recreational areas, or islands of untouched wilderness, while these sites are "are not just nodal points managing processes of pulse, flow and switch, but rather sites of amplification, elaboration and transformation”; 35 (4) dynamic spatial rhythms such as "movements, trajectories, intensities of flows of people or cars over the hour or day, groups of people starting or stopping at crossings, fluxes of people at the same speed in the same direction for identifiable period of time"; ${ }^{36}$ and (5) the rhythmic patterns of the techno-information space, which has become our artificial atmosphere, resp. mediasphere, ${ }^{37}$ where we turn on and off our computers, televisions, and mobile phones, where we are interconnected with "non-human entities into rhythmic stabilities through deployment of artefacts such as measurement devices, texts and automatic door closers ... which may routinize sequences of human acts along with coordination tools such as diaries, alarm clocks, planners that can manage the much-mooted time squeeze that exemplifies the difficulty of managing work life balance." 38

The city thus no longer constitutes an integrated whole, it has expanded and dissipated. The former, however problematic, polyrhythmic arrangements disintegrate in spams of multiple arrhythmias, collapses due to multifaceted disease. It has been overcome by congestion and intensity of its industrial rhythms enhanced up to its limits which are now melting into an indiscernible line of chaotic escape. Our current crisis which is a bundle of arrhythmic disarrangements undoubtedly reveals longer historical rhythms rhythms of gradual formation and consolidation, growth and sedimentation and subsequent decomposition and transformation of cultural formations. As any crisis, our current one brins about regrouping and transformation of all still functioning rhythms, all existing forms of permanence, on all levels of the complex system of global civilization. Today, the great closure and cessation of movement intertwines with excesses of violence, demonstrations, political and social upheavals, migrations, ecosystem collapses, global poverty, and global oversaturation.

\section{Conclusion}

The aim of this theoretical sketch was twofold: (1) to show the concept of rhythm as a complex multitude of meanings and variations; (2) to explore the scope and possibilities of current rhythmanalysis. It clearly

33 Ibid., 9.

34 See Edensor, “Introduction;” Smith and Hetherington, “Urban Rhythm;” Neuhaus, Emergent Dimensions; and Wunderlich, Symphonies.

35 Smith and Hetherington, "Urban Rhythm," 12.

36 Wunderlich, Symphonies.

37 See Lewis, Mediasphere.

38 Edensor, "Introduction," 9. 
shows that the rhythmanalysis is a potent methodological tool which allows for diverse transdisciplinary research interconnecting distinct fields of interest. This text attempts to connect ontological, phenomenological, anthropological, sociological, or ecological insights with the processual thinking and the concept of rhythm appears to be an ideal instrument to do so. Explorations presented in this article are inevitably underdeveloped, yet it was not my primary goal to accurately describe and explain the organic, garden, or urban polyrhythmic arrangements. Instead, my goal was to prepare a sketchy blueprint for further work in the field. Nevertheless, it seems indisputable that the thinking through rhythm can be at least a fruitful complement to the research of sonic environments.

Funding information: This article has been written under the support of the Czech Science Foundation grant No. 20-27355S "Phenomenological Investigations of Sonic Environments" which is solved at the Institute of Philosophy, Czech Academy of Sciences, Prague.

Conflict of interest: Author states no conflict of interest.

\section{References}

Adams, Barbara. Timescapes of Modernity. The Environment and Invisible Hazards. London/New York: Routledge, 1998.

Aristoxenus of Tarentum. Elementa Rhythmica, The Fragment of Book II and the Additional Evidence for Aristoxenean Rhythmic Theory, edited and translated by Lionel Peason, 1990.

Clayton, Kevin. "Time Folded and Crumpled: Time, History, Self-Organization and the Methodology of Michel Serres." In Time and History in Deleuze and Serres, edited by Herzogenrath, Bernd. London/NY: Continuum, 2012.

Deleuze, Gilles and Guattari, Felix. A Thousand Plateaus. Capitalism and Schizophrenia, Translated and foreword Brian Massumi. Minneapolis: University of Minnesota Press, 1987.

Edensor, Tim. “Introduction. Thinking about Rhythm and Space.” In Geographies of Rhythm. Nature, Places, Mobilities and Bodies, edited by Edensor, Tim. Farnham: Ashgate, 2010.

Eliade, Mircea. Cosmos and History. The Myth of Eternal Return. New York: Harper Torchbooks, 1954.

Gosden, Christopher. Social Being and Time. Oxford/Cambridge: Blackwell, 1994.

Grahn, Jessica A. "Neural Mechanisms of Rhythm Perception: Current Findings and Future Perspectives." Topics in Cognitive Science 4 (2012), 586-606.

Koukkari, Willard L. and Sothern, Robert B. Introducing Biological Rhythms. A Primer on the Temporal Organization of Life, with Implications for Health, Society, Reproduction and the Natural Environment. New York: Springer, 2006.

Lefebvre, Henry. Writings On Cities, edited by E. Koffman and E. Lebas. Oxford: Blackwell. 1996.

Lefebvre, Henry and Régulier, Catherine. Rhythmanalysis. Space, Time, and Everyday Life. London/NY: Continuum, 2004.

Lewis, Jeff. Crisis in Global Mediasphere. Desire, Displeasure and Cultural Transformation. New York: Palgrave Macmillan, 2011. Marcelli, Miroslav. Filozofi v Meste. Bratislava: Kalligram, 2009.

McNeill, William H. Keeping Together in Time. Dance and Drill in Human History. Cambridge/London: Harvard University Press, 1995.

Neuhaus, Fabian. Emergent Spatio-Temporal Dimensions of the City. Habitus and Urban Rhythms. Heidelberg: Springer, 2015. Palmer, John D. An Introduction to Biological Rhythms. New York/London: Academic Press, 1976.

Richir, Marc. "Pasivní Syntéze a Temporalizace/Spacializace." In Co je Fenomén? Husserl a Fenomenologie ve Francii, edited by Novotný, Karel. Praha: Oykoymenh/Pavel Mervart, 2010.

Schutz, Alfred. Fragments Toward a Phenomenology of Music. In Schutz, Alfred. Collected Papers. Volume IV. Phenomenologica 136, Springer, 1996.

Sethares, William A. Rhythm and Transforms. London: Springer, 2007.

Smith, Robin James and Hetherington, Kevin. "Urban Rhythms: Mobilities, Space and Interaction in the Contemporary City." In Urban Rhythms: Mobilities, Space and Interaction in the Contemporary City, edited by Smith, Robin James and Hetherington, Kevin. Willey Blackwell/Sociological Review, 2013.

Steiner, Rudolf. Agriculture Course. The Birth of Biodynamic Method. Rudolf Steiner Press, 2012.

The Epic of Gilgamesh. A New Translation, translated and edited by Georges, Andrew. London: Penguin Books, 1999.

Tosaki, Eiichi. Modrian's Philosophy of Visual Rhythm. Phenomenology, Wittgenstein, and Eastern Thought. Dordrecht: Springer, 2017.

Wellmann, Janine. The Form of Becoming. Embryology and Epistemology of Rhythm, 1760-1830. NY: Zone Books, 2017.

Wunderlich, Filipa Matos. Symphonies of Urban Places: Urban Rhythms as Traces of Time in Space. A Study of Urban Rhythms. In Rhutmos, 10, September 2016. Published online, available at https://rhuthmos.eu/spip.php?article1854. 